\title{
A LOWER BOUND ON THE HOMOLOGICAL BIDIMENSION OF A NON-UNITAL C*-ALGEBRA
}

\author{
by OLAF ERMERT
}

(Received 15 January, 1997)

1. Introduction. Let $A$ be a $\mathrm{C}^{*}$-algebra. For each Banach $A$-bimodule $X$, the second continuous Hochschild cohomology group $\mathcal{H}^{2}(A, X)$ of $A$ with coefficients in $X$ is defined (see [6]); there is a natural correspondence between the elements of this group and equivalence classes of singular, admissible extensions of $A$ by $X$. Specifically this means that $\mathcal{H}^{2}(A, X) \neq\{0\}$ for some $X$ if and only if there exists a Banach algebra $B$ with Jacobson radical $R$ such that $R^{2}=\{0\}, R$ is complemented as a Banach space, and $B / R \cong A$, but $B$ has no strong Wedderburn decomposition; i.e., there is no closed subalgebra $C$ of $B$ such that $B \cong C \oplus R$. In turn this is equivalent to $\mathrm{db} A \geqslant 2$, where $\mathrm{db} A$ is the homological bidimension of $A$; i.e., the homological dimension of $A^{\#}$, the unitization of $A$, as an $A$-bimodule [6, III.5.15]. This paper is concerned with the following basic question, which was posed in [7].

$$
\text { Is } \mathrm{db} A \geqslant 2 \text { for each infinite-dimensional } \mathrm{C}^{*} \text {-algebra } A \text { ? }
$$

A positive answer to this question has been obtained in each of the following cases.

(i) $A$ is commutative [8];

(ii) $A$ is separable and has a closed ideal of finite codimension that cannot be complemented as a subalgebra, or $A$ is separable and non-unital [1];

(iii) $A$ is a CCR-algebra [11].

Actually in each case the stronger result is established that $\operatorname{dg} A \geqslant 2$ in the case where $A$ is an infinite-dimensional member of the specified class; here $\operatorname{dg} A$ is the global homological dimension of $A$ [6, III.5.7], and it is known that $\operatorname{dg} A \geqslant 2$ if and only if there exist Banach left $A$-modules $Y$ and $Z$ such that $\mathcal{H}^{2}(A, \mathcal{B}(Y, Z)) \neq\{0\}$, where $\mathcal{B}(Y, Z)$ denotes the $A$-bimodule of continuous linear mappings from $Y$ into $Z$.

Let $A$ be a $C^{*}$-algebra, and suppose that $A$ admits a non-unital, closed ideal $I$ of finite codimension. We show in $\S 3$ of this paper that $\mathcal{H}^{2}(A, I \hat{\otimes} I) \neq\{0\}$ in this case, and so in particular $\mathrm{db} A \geqslant 2$. In fact, we give an explicit formula for a cocycle $\mu$ of $A$ with values in $I \hat{\otimes} I$ and show that $\mu$ does not cobound. As a corollary, we obtain the fact that $\mathrm{db} A \geqslant 2$ for each infinite-dimensional type $\mathrm{IC}^{*}$-algebra.

Finally we shall demonstrate that our methods may also be used to establish that $\operatorname{dg} A \geqslant 2$ in certain cases.

2. Preliminaries. Let $A$ be a $C^{*}$-algebra. If $A$ is unital, we write $1_{A}$ for the identity of $A$. We write $A_{+}$(respectively, $A_{\mathrm{sa}}$ ) for the positive (respectively, self-adjoint) elements of $A$, and

The author was supported by HCM grant No. ERB CHBI-CT94-1366.

Glasgow Math. J. 40 (1998) 435-444. 
we write $A^{\#}$ for the unitization of $A$ in the sense of $[12,1.1 .3]$, so that $A^{\#}=A$ if $A$ is unital, and $A^{\#}=A \oplus \mathbb{C}$ otherwise. By an approximate unit for $A$ we shall mean an increasing net of elements of $A_{+}$of norm at most one that is an approximate identity for $A$ in the usual sense. We denote by $\Lambda(A)$ the set of elements $a$ in $A_{+}$such that $\|a\|<1$. By $[12,1.4 .2], \Lambda(A)$ is an approximate unit for $A$ in the partial ordering on $A_{\text {sa }}$; as in [12], we shall refer to it as the canonical approximate unit for $A$. We denote by $S(A)$ the set of states on $A$.

Let $E$ be a Banach space. We denote by $E^{*}$ the continuous dual of $E$. Also, we write $E \otimes F$ (respectively, $E \hat{\otimes} F$ ) for the algebraic (respectiely, projective) tensor product of Banach spaces $E$ and $F$. We write $\mathcal{B}(E, F)$ for the Banach space of bounded linear maps from $E$ into $F$. The (projective) tensor product of two operators $S \in \mathcal{B}\left(E_{1}, F_{1}\right)$ and $T \in \mathcal{B}\left(E_{2}, F_{2}\right)$ is denoted by $S \otimes T \in \mathcal{B}\left(E_{1} \hat{\otimes} E_{2}, F_{1} \hat{\otimes} F_{2}\right)$.

Let $A$ be a Banach algebra. Then $A^{*}$ is a Banach $A$-bimodule for the operations

$$
\langle b, \varphi \cdot a\rangle=\langle a b, \varphi\rangle \text { and }\langle b, a \cdot \varphi\rangle=\langle b a, \varphi\rangle\left(\varphi \in A^{*}, a, b \in A\right) .
$$

Also, the Banach space $A \hat{\otimes} A$ is a Banach $A$-bimodule for the operations

$$
a \cdot(b \otimes c)=a b \otimes c \text { and }(a \otimes b) \cdot c=a \otimes b c(a, b, c \in A) .
$$

We shall use the fact that $((\varphi \cdot a) \otimes \psi)(u)=(\varphi \otimes \psi)(a \cdot u)$ and $(\varphi \otimes(a \cdot \psi))(u)=(\varphi \otimes \psi)(u \cdot a)$ for $\varphi, \psi \in A^{*}, a \in A$ and $u \in A \hat{\otimes} A$; these formulae are immediate from the definitions.

Let $A$ be a Banach algebra, and let $X$ be a Banach $A$-bimodule. We denote by $\mathcal{Z}^{2}(A, X)$ the Banach space of continuous bilinear maps $\mu: A \times A \rightarrow X$ that satisfy the cocycle identity

$$
a \cdot \mu(b, c)-\mu(a b, c)+\mu(a, b c)-\mu(a, b) \cdot c=0(a, b, c \in A) ;
$$

the elements in $\mathcal{Z}^{2}(A, X)$ are the 2-cocycles of $A$ with coefficients in $X$. For $T \in \mathcal{B}(A, X)$ we define

$$
\left(\delta^{1} T\right)(a, b)=a \cdot T(b)-T(a b)+T(a) \cdot b(a, b \in A) ;
$$

the map $T \mapsto \delta^{1} T$ is a continuous linear map from $\mathcal{B}(A, X)$ into $\mathcal{Z}^{2}(A, X)$ whose range is denoted by $\mathcal{N}^{2}(A, X)$; the elements in $\mathcal{N}^{2}(A, X)$ are the 2-coboundaries of $A$ with coefficients in $X$. The quotient group $\mathcal{Z}^{2}(A, X) / \mathcal{N}^{2}(A, X)$, denoted by $\mathcal{H}^{2}(A, X)$, is the second continuous Hochschild cohomology group of $A$ with coefficients in $X$. For more information on (Banach) Hochschild cohomology see [6] and [10]; on the question of the role of second cohomology groups and the splittings of extensions of a Banach algebra, we refer to [2].

3. Nontrivial cocycles for nonunital $\mathbf{C}^{*}$-algebras. We start this section with a general lemma, which is inspired by [6, V.2.14].

Lemma 1. Let $A$ be a $C^{*}$-algebra, let $E$ be a Banach space, and let $\Theta: A \rightarrow E \hat{\otimes} E$ be a linear map. Let $\Lambda(A)$ be the canonical approximate unit for $A$, and let $\mathcal{U}(0)$ denote the system of neighbourhoods of 0 in $\left(E^{*}, \sigma\left(E^{*}, E\right)\right.$ ). Suppose that, for each $\varepsilon>0$, there exists a non-empty subset $Y$ of the unit ball of $E^{*}$ such that, for all $\varphi_{1}, \ldots, \varphi_{n} \in Y, a \in \Lambda(A)$ and $U \in \mathcal{U}(0)$, there exist $b \in \Lambda(A)$ and $\varphi \in U \cap Y$ with $b \geqslant a$ and

$$
\left|\left(\varphi_{i} \otimes \varphi\right)(\Theta(b))-1\right|<\varepsilon, \text { for } 1 \leqslant i \leqslant n .
$$

Then $\Theta$ is unbounded. 
Proof. Assume that $\Theta$ is bounded. Let $\varepsilon>0$, and let $Y$ be the corresponding subset of the unit ball of $E^{*}$. First we inductively construct, for every positive integer $n$, elements $\varphi_{n}, \psi_{n}$ of $Y$ and $a_{n}, b_{n}$ of $\Lambda(A)$ such that the following hold for each $n$.

$$
\begin{gathered}
\left|\left(\varphi_{i} \otimes \psi_{n}\right)\left(\Theta\left(b_{n}-a_{n}\right)\right)-1\right|<2 \varepsilon \text { for } i \leqslant n ; \\
\left|\left(\varphi_{i} \otimes \psi_{j}\right)\left(\Theta\left(b_{n}-a_{n}\right)\right)\right|<\varepsilon \text { for } i \leqslant n, j<n ; \\
\left|\left(\varphi_{i} \otimes \psi_{n}\right)\left(\Theta\left(b_{j}-a_{j}\right)\right)\right|<\varepsilon \text { for } i \leqslant n, j<n ; \\
\left|\left(\varphi_{n} \otimes \psi_{i}\right)\left(\Theta\left(b_{j}-a_{j}\right)\right)\right|<\varepsilon \text { for } i, j<n ; \\
a_{n} \leqslant b_{n}, \text { and } b_{n-1} \leqslant a_{n} \text { if } n \geqslant 2 .
\end{gathered}
$$

If $n=1$, then it is immediate from the condition in the lemma that $\varphi_{1}, \psi_{1} \in Y$ and $a_{1}, b_{1} \in \Lambda(A)$ exist to satisfy (3.2) to (3.6).

Now assume that $n \geqslant 2$, and that $\varphi_{i}, \psi_{i}, a_{i}$ and $b_{i}$ have already been constructed for $1 \leqslant i \leqslant n-1$. It follows from the condition in the lemma that there exists $\varphi_{n} \in Y$ such that (3.5) is true; and we may then inductively choose elements $\rho_{1}, \rho_{2}, \ldots$ of $Y$ and an increasing sequence $x_{0}, x_{1}, \ldots$ of elements of $\Lambda(A)$ such that $x_{0}=b_{n-1}$ and

$$
\left|\left(\varphi_{i} \otimes \rho_{r}\right)\left(\Theta\left(b_{j}-a_{j}\right)\right)\right|<\varepsilon, \quad\left|\left(\varphi_{i} \otimes \rho_{r}\right)\left(\Theta\left(x_{r}-x_{r-1}\right)\right)-1\right|<2 \varepsilon
$$

for $i \leqslant n, j<n$ and $r \geqslant 1$. Since $\left(x_{r}\right)_{r}$ is an increasing sequence bounded above, we have that $x_{r}-x_{r-1} \rightarrow 0$ weakly, and so there exists $r \geqslant 1$ such that

$$
\left|\left(\varphi_{i} \otimes \psi_{j}\right)\left(\Theta\left(x_{r}-x_{r-1}\right)\right)\right|<\varepsilon
$$

for $i \leqslant n$ and $j<n$. We choose $\psi_{n}=\rho_{r}, a_{n}=x_{r-1}$ and $b_{n}=x_{r}$. Then (3.2) to (3.6) are satisfied, and the induction continues. We now fix a positive integer $n$ and consider the element $a=\sum_{i=1}^{n}\left(b_{i}-a_{i}\right)$. Certainly we
have $\|a\| \leqslant 1$. The conditions (3.2) to (3.6) imply that

$$
\left|\left(\varphi_{i} \otimes \psi_{j}\right)(\Theta(a))-1\right|<2 n \varepsilon \text { for } 1 \leqslant i \leqslant j \leqslant n
$$

and

$$
\left|\left(\varphi_{i} \otimes \psi_{j}\right)(\Theta(a))\right|<n \varepsilon \text { for } 1 \leqslant j<i \leqslant n .
$$

We may thus deduce from [6, II.2.48] (see also [9, Lemma 3.1]) that

$$
\|\Theta\| \geqslant\|\Theta(a)\| \geqslant \frac{1}{2 \pi} \log n-2 n^{2} \varepsilon
$$

which cannot be true because $\varepsilon$ and $n$ were chosen arbitrarily. We have arrived at a contradiction, and the result follows.

We can now state the main result of this paper.

Theorem 1. Let $A$ be a $\mathrm{C}^{*}$-algebra and let I be a non-unital closed ideal of $A$ of finite codimension. Then $\mathcal{H}^{2}(A, I \hat{\otimes} I) \neq 0$. In particular, $\mathrm{db} A \geqslant 2$. 
Proof. Since $\mathcal{H}^{2}(A, I \hat{\otimes} I)$ and $\mathcal{H}^{2}\left(A^{\#}, I \hat{\otimes} I\right)$ are isomorphic, we may suppose that $A$ is unital and that $A / I \neq 0$. We shall regard $A \hat{\otimes} A$ and $I \hat{\otimes} I$ as Banach $A$-bimodules in the usual way, so that $a \cdot(b \otimes c)=a b \otimes c$ and $(a \otimes b) \cdot c=a \otimes b c$ for $a, b, c \in A$. Likewise, we shall regard $(A / I) \hat{\otimes}(A / I)$ as a Banach $A / I$-bimodule. Let $\left\{e_{i j}^{k}: k=1, \ldots, N ; i, j=1, \ldots, n_{k}\right\}$ be a *-matricial basis (in the sense of [5, p. 113]) for the finite-dimensional $\mathrm{C}^{*}$-algebra $A / I$, so that $\sum_{k, i} e_{i i}^{k}=1_{A / I}$ and

$$
e_{i j}^{k} e_{s t}^{r}=\delta_{k r} \delta_{j s} e_{i t}^{k},\left(e_{i j}^{k}\right)^{*}=e_{j i}^{k}
$$

for $k, r=1, \ldots, N, i, j=1, \ldots, n_{k}$ and $s, t=1, \ldots, n_{r}$. We set

$$
\Delta=\sum_{i, k} e_{i 1}^{k} \otimes e_{1 i}^{k}
$$

and we denote by $\pi:(A / I) \hat{\otimes}(A / I) \rightarrow A / I, a \otimes b \mapsto a b$, the product map for $A / I$. Then $\Delta$ is a diagonal for $A / I$; i.e., we have $\pi(\Delta)=1$ and

$$
x \cdot \Delta=\Delta \cdot x \text { for } x \in A / I
$$

Let $\kappa: A \rightarrow A / I$ be the quotient map, and let $\rho: A / I \rightarrow A$ be a linear map such that $\kappa \circ \rho=\mathrm{id}_{A / I}$; note that $\rho$ is continuous because $A / I$ is finite-dimensional. We consider the continuous linear map

$$
T: A \rightarrow A \hat{\otimes} A: a \mapsto a \cdot(\rho \otimes \rho)(\Delta)-(\rho \otimes \rho)(\kappa(a) \cdot \Delta) .
$$

Set $\mu=\delta^{1} T$, and let $a, b \in A$. A simple calculation shows that

$$
\mu(a, b)=a \cdot(\rho \otimes \rho)(\Delta) \cdot b-a \cdot(\rho \otimes \rho)(\kappa(b) \cdot \Delta)+(\rho \otimes \rho)(\kappa(a b) \cdot \Delta)-(\rho \otimes \rho)(\kappa(a) \cdot \Delta) \cdot b .
$$

It follows that

$$
\begin{aligned}
\left(\kappa \otimes \mathrm{id}_{A}\right)(\mu(a, b))= & \kappa(a) \cdot\left(\mathrm{id}_{A / I} \otimes \rho\right)(\Delta) \cdot b-\kappa(a) \cdot\left(\mathrm{id}_{A / I} \otimes \rho\right)(\kappa(b) \cdot \Delta) \\
& +\left(\mathrm{id}_{A / I} \otimes \rho\right)(\kappa(a b) \cdot \Delta)-\left(\mathrm{id}_{A / I} \otimes \rho\right)(\kappa(a) \cdot \Delta) \cdot b \\
= & \left(\kappa(a) \cdot\left(\mathrm{id}_{A / I} \otimes \rho\right)(\Delta)-\left(\mathrm{id}_{A / I} \otimes \rho\right)(\kappa(a) \cdot \Delta)\right) \cdot b \\
& +\left(\mathrm{id}_{A / I} \otimes \rho\right)(\kappa(a b) \cdot \Delta)-\kappa(a) \cdot\left(\mathrm{id}_{A / I} \otimes \rho\right)(\kappa(b) \cdot \Delta) \\
= & 0 \cdot b+0=0 .
\end{aligned}
$$

Thus $\mu(a, b) \in \operatorname{ker}\left(\kappa \otimes \mathrm{id}_{A}\right)$. But also $\mu(a, b) \in A \otimes A$, the algebraic tensor product of $A$ with itself, and so $\mu(a, b) \in \operatorname{ker}(\kappa) \otimes A=I \otimes A$ by a standard piece of linear algebra. Analogously we can show (using (3.7)) that $\mu(a, b) \in A \otimes I$. Hence $\mu(a, b)$ lies in $(I \otimes A) \cap(A \otimes I)=I \otimes I$, and so we have shown that $\mu \in \mathcal{Z}^{2}(A, I \hat{\otimes} I)$.

We claim that $\mu$ defines a non-trivial element of the group $\mathcal{H}^{2}(A, I \hat{\otimes} I)$. To see this, let us assume that this is not the case. Then there exists a continuous linear map $\tilde{T}: A \rightarrow I \hat{\otimes} I$ such that 


$$
\mu(a, b)=a \cdot \tilde{T}(b)-\tilde{T}(a b)+\tilde{T}(a) \cdot b \quad(a, b \in A) .
$$

Recall that each $\varphi \in S(I)$ has a unique extension to a state on $A$, that we shall again denote by $\varphi$. Let $\left(u_{\lambda}\right)$ be an approximate unit for $I$, and let $\varphi, \psi \in S(I)$. From (3.8) and the definition of $\mu$, we see that

$$
u_{\lambda} \cdot(\rho \otimes \rho)(\Delta) \cdot u_{\mu}=u_{\lambda} \cdot \tilde{T}\left(u_{\mu}\right)-\tilde{T}\left(u_{\lambda} u_{\mu}\right)+\tilde{T}\left(u_{\lambda}\right) \cdot u_{\mu}
$$

and therefore

$$
\begin{aligned}
\left(\left(\varphi \cdot u_{\lambda}\right) \otimes\left(u_{\mu} \cdot \psi\right)\right)((\rho \otimes \rho)(\Delta))= & \left(\left(\varphi \cdot u_{\lambda}\right) \otimes \psi\right)\left(\tilde{T}\left(u_{\mu}\right)\right)-(\varphi \otimes \psi)\left(\tilde{T}\left(u_{\lambda} u_{\mu}\right)\right) \\
& +\left(\varphi \otimes\left(u_{\mu} \cdot \psi\right)\right)\left(\tilde{T}\left(u_{\lambda}\right)\right)
\end{aligned}
$$

for all $\lambda, \mu$. Taking limits, first over $\lambda$ (for fixed $\mu$ ) and then over $\mu$, and using the fact that the nets $\left(\varphi \cdot u_{\lambda}\right)$ (respectively, $\left.\left(u_{\lambda} \cdot \psi\right)\right)$ are norm-convergent to $\varphi$ (respectively, to $\psi$ ), we obtain that

$$
(\varphi \otimes \psi)((\rho \otimes \rho)(\Delta))=\lim _{\lambda}(\varphi \otimes \psi)\left(\tilde{T}\left(u_{\lambda}\right)\right) .
$$

Since $I$ is non-unital, it admits a net $\left(\varphi_{\alpha}\right)$ of states that is weak-* convergent to $0[4,2.12 .13]$. We may suppose that $\left(\varphi_{\alpha}\right)$ converges in the weak-* topology on $A^{*}$ to a state $\varphi$ on $A$. Then $\varphi$ vanishes on $I$, and $\varphi \circ \rho$ is a state on $A / I$. Hence there exists a unitary element $u$ in $A / I$ and $1 \leqslant l \leqslant N$ such that $\varphi\left(\rho\left(u e_{11}^{l}\right) \rho\left(u e_{11}^{\prime}\right)^{*}\right)=\varphi\left(\rho\left(u e_{11}^{l} u^{*}\right)\right)>0$; we may suppose that $\varphi_{\alpha}\left(\rho\left(u e_{11}^{l}\right) \rho\left(u e_{11}^{l}\right)^{*}\right) \geqslant \delta>0$, for some $\delta$ independent of $\alpha$. We consider the positive functionals

$$
\psi_{\alpha}: I \rightarrow \mathbb{C}: a \mapsto\left(\varphi_{\alpha}\left(\rho\left(u e_{11}^{l}\right) \rho\left(u e_{11}^{l}\right)^{*}\right)\right)^{-1} \varphi_{\alpha}\left(\rho\left(u e_{11}^{l}\right) a \rho\left(u e_{11}^{l}\right)^{*}\right) .
$$

Let $\left(u_{\lambda}\right)$ be an approximate identity for $I$ which is quasi-central (see [12, 3.12.14]), so that $\left\|u_{\lambda} a-a u_{\lambda}\right\| \rightarrow 0$, for each $a \in A$. Then it is easily checked that $\lim _{\lambda} \psi_{\alpha}\left(u_{\lambda}\right)=1$, and so each $\psi_{\alpha}$ is a state on $I$. Clearly we have

$$
\psi_{\alpha} \rightarrow 0\left(\text { in } \sigma\left(I^{*}, I\right)\right) \text { and } \psi_{\alpha}\left(\rho\left(e_{11}^{l}\right)\right) \rightarrow 1 .
$$

Now let $\Theta$ be the restriction of $\tilde{T}$ to $I$, and let $\Lambda(I)$ be the canonical approximate unit for $I$. We wish to apply Lemma 1 to $\Theta$, and so we choose $\varepsilon>0$. Let $Y$ be the set of $\varphi$ in $S(I)$ such that $\left|\varphi\left(\rho\left(e_{11}^{l}\right)\right)-1\right|<\varepsilon$. Choose $\varphi_{1}, \ldots, \varphi_{n} \in Y, a \in \Lambda(I)$ and a neighbourhood $U$ of 0 in $\left(I^{*}, \sigma\left(I^{*}, I\right)\right.$ ). From (3.10) we see that we can find $\alpha_{0}$ such that $\psi_{\alpha} \in Y \cap U$ for each $\alpha \geqslant \alpha_{0}$; it also follows that the $\psi_{\alpha}$, considered as functionals on $A$, are weak-* convergent to the state $\psi \circ \kappa$, where $\psi$ is the pure state on $A / I$ that satisfies $\psi\left(e_{11}^{l}\right)=1$ and $\psi\left(e_{i j}^{k}\right)=0$ whenever $(k, i, j) \neq(l, 1,1)$. Let $\varphi \in S(I)$. Then

$$
\begin{aligned}
\lim _{\alpha}\left(\varphi \otimes \psi_{\alpha}\right)((\rho \otimes \rho)(\Delta)) & =(\varphi \otimes \psi \circ \kappa)((\rho \otimes \rho)(\Delta)) \\
& =(\varphi \otimes \psi)((\rho \otimes \mathrm{id})(\Delta)) \\
& =\sum_{k, i} \varphi\left(\rho\left(e_{i 1}^{k}\right)\right) \psi\left(e_{1 i}^{k}\right)=\varphi\left(\rho\left(e_{11}^{l}\right)\right) .
\end{aligned}
$$


Hence, by (3.9) and the definition of $Y$, we may choose $\alpha_{1} \geqslant \alpha_{0}$ and then $b \in \Lambda(I)$ such that $b \geqslant a$ and

$$
\left|\left(\varphi_{i} \otimes \psi_{\alpha_{1}}\right)(\Theta(b))-1\right|<\varepsilon \quad(1 \leqslant i \leqslant n)
$$

Choose $\varphi=\psi_{\alpha_{1}}$. Then $\varphi \in Y \cap U$, and the condition (3.1) of Lemma 1 is satisfied. Consequently $\Theta$ is unbounded. This is a contradiction.

REMARK. In the case where $A$ is non-unital and $I=A$, the cocycle that we have constructed is the map $\mu:(a, b) \mapsto a \otimes b, A \times A \rightarrow A \hat{\otimes} A$. This cocycle has been considered before in [9, Theorem 3.2], where it was shown that $\mu$ defines a non-trivial element of $\mathcal{H}^{2}(A, A \hat{\otimes} A)$ in the case where $A$ is a non-unital, amenable Banach function algebra. It would be interesting to know whether the condition that $A$ be amenable is needed here.

We shall need the fact that each infinite-dimensional type $\mathrm{IC}^{*}$-algebra contains a closed non-unital ideal of finite codimension. A proof of this is (implicitly) contained in [1, §7]; we shall give another, shorter, proof here for the sake of completeness.

Recall that a closed ideal $I$ of a $C^{*}$-algebra $A$ is essential $([12,3.12 .7])$ if each non-zero closed ideal of $A$ has non-zero intersection with $I$, or, equivalently, when the annihilator $I^{\perp}=\{a \in A: a I=0\}$ is zero. If $I$ is unital, then $I^{\perp}=\left(1_{A^{\#}}-1_{I}\right) A$ and $I \oplus I^{\perp}=A$; hence if $I$ is unital and essential, then $I=A$.

Corollary 1. Let $A$ be an infinite-dimensional type I $\mathrm{C}^{*}$-algebra. Then $\mathrm{db} A \geqslant 2$.

Proof. Let $\mathcal{C}$ be the set of non-unital closed ideals of $A$. An application of Zorn's lemma shows that the set $\mathcal{C} \cup\{0\}$ has a maximal element, $I$ say. Set $B=A / I$. By $[12,6.2 .11], B$ contains a closed, essential ideal $J$ which has continuous trace. The maximality of $I$ implies that $J$ is unital. Thus $J=B$ and $B$ has continuous trace. Hence by $[12,6.1 .11]$ the primitive ideal space $\operatorname{Prim}(B)$ of $B$ is a compact Hausdorff space. Let $P \in \operatorname{Prim}(B)$, and let $F=\operatorname{Prim}(B) \backslash\{P\}$. Then $F$ is homeomorphic to $\operatorname{Prim}(P)$. But $P$ is unital, and so (see $[4,3.1 .8]$ ) Prim $(P)$ is compact. Hence $F$ is a compact, and therefore closed, subset of $\operatorname{Prim}(B)$. We have shown that $\operatorname{Prim}(B)$ is a discrete compact space, which must therefore be finite. Thus $B=A / I$ is finitedimensional. The result now follows from Theorem 1 .

Let $I$ be a closed ideal of a $C^{*}$-algebra $A$, and let $X$ be a Banach $A / I$-bimodule. Then the quotient map $\kappa: A \rightarrow A / I$ induces a Banach $A$-bimodule structure on $X$. We have a canonical map

$$
\mathcal{H}^{2}(A / I, X) \rightarrow \mathcal{H}^{2}(A, X), \mu+\mathcal{N}^{2}(A / I, X) \mapsto \mu \circ(\kappa \otimes \kappa)+\mathcal{N}^{2}(A, X) .
$$

We claim that this is an embedding. Indeed, let $\mu \in \mathcal{Z}^{2}(A / I, X)$, and suppose that $\mu \circ(\kappa \otimes \kappa) \epsilon$ $\mathcal{N}^{2}(A, X)$. Then there exists a continuous linear map $T: A \rightarrow X$ such that

$$
\mu(a+I, b+I)=(a+I) \cdot T(b)-T(a b)+T(a) \cdot(b+I) \quad(a, b \in A) .
$$

It follows that $T$ vanishes on $I^{2}$, the set of all products of two elements of $I$. But $I^{2}=I$ by Cohen's factorization theorem. Thus $I \subseteq \operatorname{ker} T$ and $T$ induces a continuous linear map $\tilde{T}: A / I \rightarrow X$. Clearly we have $\mu=\delta^{1} \tilde{T}$. Hence $\mu \in \mathcal{N}^{2}(A / I, X)$, and our claim follows.

In particular, we see that $\mathrm{db} A \geqslant 2$ if $\mathrm{db} A / I \geqslant 2$. This fact together with an easy adaptation of the proof of [1, Theorem 4] implies that the following theorem analogous to [1, Theorem 4] is true. 
THEOREM 2. Suppose that $\mathrm{db} A \geqslant 2$ for each infinite-dimensional, unital, simple $\mathrm{C}^{*}$-algebra $A$. Then $\mathrm{db} A \geqslant 2$, for each infinite-dimensional $\mathrm{C}^{*}$-algebra $A$.

Although primarily designed to give lower bounds on $\mathrm{db} A$, our methods may also be used to establish that $\operatorname{dg} A \geqslant 2$ in certain cases. We shall demonstrate this in our next theorem, where we give another application of Lemma 1.

For Banach left $A$-modules $Y$ and $Z$, the Banach space $\mathcal{B}(Y, Z)$ will always be endowed with the $A$-bimodule structure given by

$$
(a \cdot T)(y)=a \cdot T(y) \text { and }(T \cdot b)(y)=T(b \cdot y) \quad(y \in Y)
$$

for $a, b \in A$ and $T \in \mathcal{B}(Y, Z)$. From the general theory it is known that $\operatorname{dg} A \geqslant 2$ if and only if $\mathcal{H}^{2}(A, \mathcal{B}(Y, Z)) \neq\{0\}$, for some $Y$ and $Z$. (See [6, III.5.15].)

TheOREM 3. Let $A$ be a $\mathrm{C}^{*}$-algebra and let I be a closed ideal of $A$ of finite codimension. Suppose that I admits a sequence of states that is weak-* convergent to 0 . Then we have $\mathcal{H}^{2}\left(A, \mathcal{B}\left(I^{* *}, I \hat{\otimes}\left(I \cdot I^{* *}\right)\right)\right) \neq\{0\}$. In particular, $\operatorname{dg} A \geqslant 2$.

Remarks. (i) Here $I \cdot I^{* *}$ denotes the set of all products $a b$, where $a \in I$ and $b \in I^{* *}$. By the Cohen factorization theorem, $I \cdot I^{* *}$ is a closed subspace of $I^{* *}$.

(ii) We shall regard $I^{* *}$ and $I \hat{\otimes}\left(I \cdot I^{* *}\right)$ as left Banach $A$-modules in the usual way, so that $\mathcal{B}\left(I^{* *}, I \hat{\otimes}\left(I \cdot I^{* *}\right)\right)$ carries the $A$-bimodule structure defined in (3.11).

(iii) Clearly the condition on $I$ in the theorem is satisfied in the case where $I$ is non-unital and separable. Hence our theorem contains the main result in [1].

Proof. Suppose that $I$ satisfies the condition in the theorem. Let $\mu: A \times A \rightarrow I \hat{\otimes} I$ be the cocycle constructed in Theorem 1 . For $a, b \in A$ and $c \in I^{* *}$ we set

$$
\nu(a, b)(c)=\mu(a, b) \cdot c .
$$

This defines a map $v: A \times A \rightarrow \mathcal{B}\left(I^{* *}, I \hat{\otimes}\left(I \cdot I^{* *}\right)\right)$, and it is easily checked that $v$ is 2-cocycle. We claim that $\nu$ defines a non-trivial element of the group $\mathcal{H}^{2}\left(A, \mathcal{B}\left(I^{* *}, I \hat{\otimes}\left(I \cdot I^{* *}\right)\right)\right)$. To see this, let us assume that this is not the case. Then there is a continuous linear map $T: A \rightarrow \mathcal{B}\left(I^{* *}, I \hat{\otimes}\left(I \cdot I^{* *}\right)\right)$ such that

$$
a \cdot T(b)(c)-T(a b)(c)+T(a)(b c)=\mu(a, b) \cdot c
$$

for all $a, b \in A$ and $c \in I^{* *}$. We set $E=I \cdot I^{* *}$, and for $a \in I$ we set

$$
\Theta(a)=\left(\iota \otimes \mathrm{id}_{E}\right)\left(T(a)\left(1_{I^{*}}\right)\right),
$$

where $\iota: I \hookrightarrow E$ is the inclusion map. Then $\Theta: I \rightarrow E \hat{\otimes} E$ is a continuous linear map. We wish to apply Lemma 1 to $\Theta$. Let $\varepsilon>0$ be given.

As in Theorem 1, let $\rho: A / I \rightarrow A$ be a linear map that is a right inverse for the quotient map $\kappa: A \rightarrow A / I$. The condition on $I$ implies that the construction in the proof of Theorem 1 yields a *-matrical basis $\left\{e_{i j}^{k}\right\}$ of $A / I$ and a sequence $\left(\psi_{m}\right)$ of states on $I$ that is weak-* convergent to 0 such that

$$
\lim _{m}\left(\psi \otimes \psi_{m}\right)((\rho \otimes \rho)(\Delta))=\psi\left(\rho\left(e_{11}^{1}\right)\right) \quad(\psi \in S(I))
$$


where $\Delta=\sum_{k, i} e_{i 1}^{k} \otimes e_{1 i}^{k}$, and

$$
\lim _{m} \psi_{m}\left(\rho\left(e_{11}^{1}\right)\right)=1
$$

Let $Y_{0}$ be the set of all $\phi \in S(I)$ such that $\left|\phi\left(\rho\left(e_{11}^{1}\right)\right)-1\right|<\varepsilon$. Let $\alpha: I^{*} \hookrightarrow I^{* * *}$ be the canonical embedding, and let $\beta: I^{* * *} \rightarrow E^{*}$ be the map that restricts an element $\psi$ in $I^{* * *}$ to $E$. We choose $Y$ to be the image of $Y_{0}$ under the mapping $\beta \circ \alpha: I^{*} \rightarrow E^{*}$. Choose $\varphi_{1}, \ldots, \varphi_{n} \in Y, a \in \Lambda(I)$ and a neighbourhood $U$ of 0 in $\left(E^{*}, \sigma\left(E^{*}, E\right)\right)$. By the definition of $Y$, there are $\phi_{1}, \ldots, \phi_{n} \in Y_{0}$ such that $\varphi_{i}=(\beta \circ \alpha)\left(\phi_{i}\right),(i=1, \ldots, n)$. For each $m$ we set $\tau_{m}=(\beta \circ \alpha)\left(\psi_{m}\right)$. Clearly each $\tau_{m}$ is an extension of $\psi_{m}$ to $E$ and it is easily checked that $\tau_{m} \rightarrow 0$ in the weak-* topology on $E^{*}$. Hence, by (3.14) there exists $m_{0}$ such that $\tau_{m} \in Y \cap U$, for all $m \geqslant m_{0}$. Also it follows from (3.13) and the definition of $Y$ that there exist $\delta \in(0, \varepsilon), c \in \Lambda(I)$ and $m_{1} \geqslant m_{0}$ such that

$$
\left|\left(\left(\phi_{i} \cdot c\right) \otimes \psi_{m}\right)((\rho \otimes \rho)(\Delta))-1\right| \leqslant \varepsilon-\delta \quad(1 \leqslant i \leqslant n),
$$

for all $m \geqslant m_{1}$, and

$$
\left\|\phi_{i} \cdot c-\phi_{i}\right\|\|\Theta\|<\delta \quad(1 \leqslant i \leqslant n)
$$

Now let $u_{0}=0 \leqslant u_{1} \leqslant u_{2} \ldots$ be an increasing sequence in $\Lambda(I)$ such that $u_{1} \geqslant a$ and

$$
\lim _{m}\left\|c u_{m}-c\right\|=0 \text { and } \lim _{m} \psi_{m}\left(u_{m}\right)=1 .
$$

By (3.12) and the definition of $\mu$ we have that

$$
c \cdot \Theta\left(u_{m}\right)-\Theta\left(c u_{m}\right)+T(c)\left(u_{m}\right)=\mu\left(c, u_{m}\right)=c \cdot(\rho \otimes \rho)(\Delta) \cdot u_{m}
$$

for all $m$. Hence by (3.15)

$$
\begin{aligned}
\left|\left(\left(\varphi_{i} \cdot c\right) \otimes \tau_{m}\right)\left(\Theta\left(u_{m}\right)\right)-1\right| \leqslant & \left|\left(\left(\phi_{i} \cdot c\right) \otimes\left(\left(1_{I^{* *}}-u_{m}\right) \cdot \psi_{m}\right)\right)((\rho \otimes \rho)(\Delta))\right| \\
& +\left|\left(\varphi_{i} \otimes \tau_{m}\right)\left(\Theta\left(c u_{m}\right)\right)\right|+\left|\left(\phi_{i} \otimes \tau_{m}\right)\left(T(c)\left(u_{m}\right)\right)\right|+\varepsilon-\delta
\end{aligned}
$$

for all $i \in\{1, \ldots, n\}$ and $m \geqslant m_{1}$. We have that

$$
\left|\left(\varphi_{i} \otimes \tau_{m}\right)\left(\Theta\left(c u_{m}\right)\right)\right| \leqslant\left|\left(\varphi_{i} \otimes \tau_{m}\right)(\Theta(c))\right|+\|\Theta\|\left\|c u_{m}-c\right\|
$$

for all $m$. But $\Theta(c) \in E \hat{\otimes} E$ and consequently

$$
\lim _{m}\left|\left(\varphi_{i} \otimes \tau_{m}\right)\left(\Theta\left(c u_{m}\right)\right)\right|=0 \quad(1 \leqslant i \leqslant n) .
$$

Also we have $\left\|\left(1_{I^{* *}}-u_{m}\right) \cdot \psi_{m}\right\| \leqslant\left(1-\psi_{m}\left(u_{m}\right)\right)^{\frac{1}{2}} \rightarrow 0$ as $m \rightarrow \infty$ and therefore

$$
\lim _{m}\left|\left(\left(\phi_{i} \cdot c\right) \otimes\left(\left(1_{l^{* *}}-u_{m}\right) \cdot \psi_{m}\right)\right)((\rho \otimes \rho)(\Delta))\right|=0
$$

for all $i \in\{1, \ldots, n\}$. 
For $\lambda=\left(\lambda_{j}\right) \in c_{0}(\mathbb{N})$ we set $\sigma(\lambda)=\sum_{j=1}^{\infty} \lambda_{j}\left(u_{j}-u_{j-1}\right)$. It is straightforward to check that $\sigma$ is a well-defined continuous linear map from $c_{0}(\mathbb{N})$ into $I$. Therefore $\sigma^{* *}$ maps $l^{\infty}(\mathbb{N})$ into $I^{* *}$. Fix $\varphi \in S(I)$. For each positive integer $m$ we define

$$
\mathcal{X}_{m}: P^{\infty}(\mathbb{N}) \rightarrow \mathbb{C}: \lambda \mapsto\left(\varphi \otimes \tau_{m}\right)\left(T(c)\left(\sigma^{* *}(\lambda)\right)\right)
$$

Then $\left(\mathcal{X}_{m}\right)$ is a sequence of continuous functionals on $l^{\infty}(\mathbb{N})$ that is weak-* convergent to 0 . By Phillips's lemma, we have that $\lim _{m} \sum_{j=1}^{\infty}\left|\mathcal{X}_{m}\left(e_{j}\right)\right|=0$, where $e_{j}$ is the sequence which has 1 in the $j$-th position and 0 elsewhere. (For a proof see $[3, \mathrm{p} .83]$; note that this lemma has been used, in similar situations, in [6, V.2.15] and also [1].) But

$$
\left|\left(\varphi \otimes \tau_{m}\right)\left(T(c)\left(u_{m}\right)\right)\right| \leqslant \sum_{j=1}^{m} \mid\left(\varphi \otimes \tau_{m}\right)\left(T(c)\left(u_{j}-u_{j-1}\right)\left|=\sum_{j=1}^{m}\right| \mathcal{X}_{m}\left(e_{j}\right)\left|\leqslant \sum_{j=1}^{\infty}\right| \mathcal{X}_{m}\left(e_{j}\right) \mid\right.
$$

for all $m$, and so we conclude that $\lim _{m}\left|\left(\varphi \otimes \tau_{m}\right)\left(T(c)\left(u_{m}\right)\right)\right|=0$. This together with (3.16), (3.17), (3.18) and (3.19) shows that there exists $m_{2} \geqslant m_{1}$ such that

$$
\left|\left(\varphi_{i} \otimes \tau_{m_{2}}\right)\left(\Theta\left(u_{m_{2}}\right)\right)-1\right| \leqslant \varepsilon \text { for } 1 \leqslant i \leqslant n
$$

We now choose $\varphi=\tau_{m_{2}}$ and $b=u_{m_{2}}$. Then the condition (3.1) in Lemma 1 is satisfied, and so by this lemma $\Theta$ is unbounded. This is a contradiction.

Acknowledgements. The author would like to thank Professor H.G. Dales, Dr. Z. Lykova and Dr. O. Aristov for stimulating and helpful discussions on the subject of this note.

\section{REFERENCES}

1. O. Yu. Aristov, The global dimension theorem for non-unital and some other separable $\mathrm{C}^{*}$ algebras, Mat. Sbornik 186 (1995), 3-18=Russ. Acad. Sci. Sb. Math. 186 (1995), 1223-1229.

2. W. G. Bade, H. G. Dales and Z. A. Lykova, Algebraic and strong splittings of extensions of Banach algebras, Mem. American Math. Soc., to appear.

3. J. Diestel, Sequences and series in Banach spaces, Graduate Texts in Mathematics 92 (SpringerVerlag, New York, 1984).

4. J. Dixmier, C*-algebras (North-Holland, 1977). 1982).

5. K. R. Goodearl, Notes on real and complex $C^{*}$-algebras (Shiva Publishing Ltd. Nantwich,

6. A. Ya. Helemskii, The homology of Banach and topological algebras (Kluwer Academic Publishers, 1986).

7. A. Ya. Helemskii, 31 problems of the homology of the algebras of analysis in Linear complex analysis problem book, Part I, Lecture Notes in Mathematics No. 1573, (Springer-Verlag, 1994), 54-78.

8. A. Ya. Helemskii, The global dimension of a Banach function algebra is different from one, Funct. anal. i pril. 6 (1972), 95-96= Functional Anal. Appl. 6 (1972), 166-168.

9. B. E. Johnson, Approximate diagonals and cohomology of certain annihilator Banach algebras, Amer. J. Math. 94 (1972), 685-698.

10. B. E. Johnson, Cohomology in Banach algebras, Mem. American Math. Soc. 127 (1972). 
11. Z. A. Lykova, The lower estimate of the global homological dimension of infinite-dimensional CCR algebras, in Operator algebras and topology (Proceedings of the OATE 2 Conference, Roumania, 1989), Pitman Research Notes in Mathematics, No. 270, (Longman, 1992), 93-129.

12. G. K. Pederson, $C^{*}$-algebras and their automorphism groups (Academic Press, London, 1979).

SChOOL OF Mathematics

UNIVERSITY OF LEEDS

LEEDS LS2 9JT

ENGLAND
Present address:

FACHBEREICH MATHEMATIK

UNIVERSITÄT DES SAARLANDES

D-66041 SAARBRÜCKEN

GERMANY

Email: ermert@math.uni-sb.de 